\title{
A Marcinkiewicz-Zygmund type strong law of large numbers for non-negative random variables with multidimensional indices
}

\author{
Tibor Tómács* \\ Institute of Mathematics and Informatics \\ Eszterházy Károly University, Eger, Hungary \\ tomacs.tibor@uni-eszterhazy.hu \\ Submitted: September 2, 2019 \\ Accepted: December 4, 2019 \\ Published online: December 5, 2019
}

\begin{abstract}
In this paper a Marcinkiewicz-Zygmund type strong law of large numbers is proved for non-negative random variables with multidimensional indices, furthermore we give its an application for multi-index sequence of nonnegative random variables with finite variances.
\end{abstract}

Keywords: Marcinkiewicz-Zygmund type strong law of large numbers, almost sure convergence, non-negative random variables, multidimensional indices

$M S C: 60 \mathrm{~F} 15$

\section{Introduction}

The Kolmogorov theorem and the Marcinkiewicz-Zygmund theorem are two famous theorems on the strong law of large numbers for $X_{n}(n \in \mathbb{N})$ sequence of independent identically distributed random variables (see e.g. LoÈvE [8]). By Kolmogorov theorem, there exists a constant $b$ such that $\lim _{n \rightarrow \infty} S_{n} / n=b$ almost surely if and only if $\mathrm{E}\left|X_{1}\right|<\infty$, where $S_{n}=\sum_{k=1}^{n} X_{k}$. If the latter condition is satisfied then $b=\mathrm{E} X_{1}$. By Marcinkiewicz-Zygmund theorem, if $0<r<2$ then

\footnotetext{
*The author's research was supported by the grant EFOP-3.6.1-16-2016-00001 ("Complex improvement of research capacities and services at Eszterhazy Karoly University").
} 
$\lim _{n \rightarrow \infty}\left(S_{n}-b n\right) / n^{1 / r}=0$ almost surely if and only if $\mathrm{E}\left|X_{1}\right|^{r}<\infty$, where $b=0$ if $0<r<1$, and $b=\mathrm{E} X_{1}$ if $1 \leq r<2$.

ETEmadi [1] proved that the Kolmogorov theorem holds for identically distributed and pairwise independent random variables, furthermore KRUGLOV [7] extended the Marcinkiewicz-Zygmund theorem for pairwise independent case if $r<1$.

Several papers are devoted to the study of the strong law of large numbers for multi-index sequence of random variables (see e.g. Gut [4], KLESOv [5, 6], FAZEKAS [2], Fazekas, Tómács [3]). For example, Theorem 3.1 of Fazekas, Tómács [3] extends Theorem 2 of KRUGLOV [7] for multi-index case.

In this paper the main result is Theorem 3.1, which is a Marcinkiewicz-Zygmund type strong law of large numbers for non-negative random variables with multidimensional indices. It is a generalization of Theorem 3.1 of FAZEKAS, TómÁcs [3] in case $\mathbf{n} \rightarrow \infty$. Furthermore we give an application (see Theorem 4.1) for multiindex sequence of non-negative random variables with finite variances. A special case of this result gives Theorem of PeTrov [9].

\section{Notation}

Let $\mathbb{N}^{d}$ be the positive integer $d$-dimensional lattice points, where $d$ is a positive integer. For $\mathbf{n}, \mathbf{m} \in \mathbb{N}^{d}, \mathbf{n} \leq \mathbf{m}$ is defined coordinate-wise, $(\mathbf{n}, \mathbf{m}]=\left(n_{1}, m_{1}\right] \times$ $\left(n_{2}, m_{2}\right] \times \cdots \times\left(n_{d}, m_{d}\right]$ is a $d$-dimensional rectangle and $|\mathbf{n}|=n_{1} n_{2} \cdots n_{d}$, where $\mathbf{n}=\left(n_{1}, n_{2}, \ldots, n_{d}\right), \mathbf{m}=\left(m_{1}, m_{2}, \ldots, m_{d}\right) . \sum_{\mathbf{n}}$ will denote the summation for all $\mathbf{n} \in \mathbb{N}^{d}$. We also use $\mathbf{1}=(1,1, \ldots, 1) \in \mathbb{N}^{d}$ and $\mathbf{2}=(2,2, \ldots, 2) \in \mathbb{N}^{d}$. Denote the integer part of $x$ real number by $[x]$.

We shall say that $\lim _{\mathbf{n} \rightarrow \infty} a_{\mathbf{n}}=0$, where $a_{\mathbf{n}}\left(\mathbf{n} \in \mathbb{N}^{d}\right)$ are real numbers, if for all $\delta>0$ there exists $\mathbf{N} \in \mathbb{N}^{d}$ such that $\left|a_{\mathbf{n}}\right|<\delta \forall \mathbf{n} \geq \mathbf{N}$.

We shall assume that random variables $X_{\mathbf{n}}\left(\mathbf{n} \in \mathbb{N}^{d}\right)$ are defined on the same probability space $(\Omega, \mathcal{F}, \mathrm{P})$. E and Var stand for the expectation and the variance.

Remark that a sum or a minimum over the empty set will be interpreted as zero (i.e. $\sum_{\mathbf{n} \in H} a_{\mathbf{n}}=\min _{\mathbf{n} \in H} a_{\mathbf{n}}=0$ if $H=\emptyset$ ).

\section{The result}

The following result is a generalization of Theorem 3.1 of FAzEKAS, TómÁcs [3] in case $\mathbf{n} \rightarrow \infty$.

Theorem 3.1. Let $X_{\mathbf{n}}\left(\mathbf{n} \in \mathbb{N}^{d}\right)$ be a sequence of non-negative random variables, let $b_{\mathbf{n}}\left(\mathbf{n} \in \mathbb{N}^{d}\right)$ be a sequence of non-negative numbers, $B_{\mathbf{n}}=\sum_{\mathbf{k} \leq \mathbf{n}} b_{\mathbf{k}}, S_{\mathbf{n}}=$ $\sum_{\mathbf{k} \leq \mathbf{n}} X_{\mathbf{k}}, c>0, K \in \mathbb{N}$ and $0<r \leq 1$. If

$$
B_{\mathbf{n}}-B_{\mathbf{m}} \leq c(|\mathbf{n}|-|\mathbf{m}|) \quad \forall \mathbf{n}, \mathbf{m} \in \mathbb{N}^{d}, \mathbf{n} \geq \mathbf{m},|\mathbf{n}|-|\mathbf{m}| \geq K
$$


and

$$
\sum_{\mathbf{n}} \frac{1}{|\mathbf{n}|} \mathrm{P}\left(\left|S_{\mathbf{n}}-B_{\mathbf{n}}\right|>\varepsilon|\mathbf{n}|^{1 / r}\right)<\infty \quad \forall \varepsilon>0
$$

then

$$
\lim _{\mathbf{n} \rightarrow \infty} \frac{S_{\mathbf{n}}-B_{\mathbf{n}}}{|\mathbf{n}|^{1 / r}}=0 \quad \text { almost surely. }
$$

Proof. Let $\delta>0,1<\alpha<\left(\frac{\delta}{2 c}+1\right)^{1 / 3 d}$ and $0<\varepsilon<\frac{\delta}{2}\left(\frac{\delta}{2 c}+1\right)^{-1 / r}$, which imply

$$
\varepsilon \alpha^{3 d / r}+c\left(\alpha^{3 d}-1\right)<\delta .
$$

Let $k_{n}=\left[\alpha^{n}\right](n \in \mathbb{N})$ and $\mathbf{k}_{\mathbf{n}}=\left(k_{n_{1}}, k_{n_{2}}, \ldots, k_{n_{d}}\right)$, where $\mathbf{n}=\left(n_{1}, n_{2}, \ldots, n_{d}\right) \in$ $\mathbb{N}^{d}$. It follows from the inequalities

$$
\begin{aligned}
& \sum_{\mathbf{n}} \frac{1}{|\mathbf{n}|} \mathrm{P}\left(\left|S_{\mathbf{n}}-B_{\mathbf{n}}\right|>\varepsilon|\mathbf{n}|^{1 / r}\right) \\
& \geq \sum_{\mathbf{n}} \sum_{\mathbf{h} \in\left(\mathbf{k}_{\mathbf{n}}, \mathbf{k}_{\mathbf{n}+\mathbf{1}}\right]} \frac{1}{|\mathbf{h}|} \mathrm{P}\left(\left|S_{\mathbf{h}}-B_{\mathbf{h}}\right|>\varepsilon|\mathbf{h}|^{1 / r}\right) \\
& \geq \sum_{\mathbf{n}} \sum_{\mathbf{h} \in\left(\mathbf{k}_{\mathbf{n}}, \mathbf{k}_{\mathbf{n}+\mathbf{1}}\right]} \frac{1}{\left|\mathbf{k}_{\mathbf{n}+\mathbf{1}}\right|} \min _{\mathbf{k} \in\left(\mathbf{k}_{\mathbf{n}}, \mathbf{k}_{\mathbf{n}+\mathbf{1}}\right]} \mathrm{P}\left(\left|S_{\mathbf{k}}-B_{\mathbf{k}}\right|>\varepsilon|\mathbf{k}|^{1 / r}\right) \\
& =\sum_{\mathbf{n}} \frac{\left|\mathbf{k}_{\mathbf{n}+\mathbf{1}}-\mathbf{k}_{\mathbf{n}}\right|}{\left|\mathbf{k}_{\mathbf{n}+\mathbf{1}}\right|} \min _{\mathbf{k} \in\left(\mathbf{k}_{\mathbf{n}}, \mathbf{k}_{\mathbf{n}+\mathbf{1}}\right]} \mathrm{P}\left(\left|S_{\mathbf{k}}-B_{\mathbf{k}}\right|>\varepsilon|\mathbf{k}|^{1 / r}\right)
\end{aligned}
$$

and condition (3.2) that

$$
\sum_{\mathbf{n}} \frac{\left|\mathbf{k}_{\mathbf{n}+\mathbf{1}}-\mathbf{k}_{\mathbf{n}}\right|}{\left|\mathbf{k}_{\mathbf{n}+\mathbf{1}}\right|} \min _{\mathbf{k} \in\left(\mathbf{k}_{\mathbf{n}}, \mathbf{k}_{\mathbf{n}+\mathbf{1}}\right]} \mathrm{P}\left(\left|S_{\mathbf{k}}-B_{\mathbf{k}}\right|>\varepsilon|\mathbf{k}|^{1 / r}\right)<\infty .
$$

Since $\lim _{n \rightarrow \infty}\left(1-\frac{1}{\alpha^{n+1}}-\frac{1}{\alpha}\right)=1-\frac{1}{\alpha}>0$, so $\left(1-\frac{1}{\alpha^{n+1}}-\frac{1}{\alpha}\right)>\frac{\alpha-1}{2 \alpha}$ except for finitely many $n \in \mathbb{N}$. This implies that there exists $\mathbf{N}_{0} \in \mathbb{N}^{d}$ such that

$$
\begin{aligned}
0<\left(\frac{\alpha-1}{2 \alpha}\right)^{d} & <\prod_{i=1}^{d}\left(1-\frac{1}{\alpha^{n_{i}+1}}-\frac{1}{\alpha}\right)=\prod_{i=1}^{d} \frac{\alpha^{n_{i}+1}-1-\alpha^{n_{i}}}{\alpha^{n_{i}+1}} \\
& \leq \prod_{i=1}^{d} \frac{\left[\alpha^{n_{i}+1}\right]-\left[\alpha^{n_{i}}\right]}{\left[\alpha^{n_{i}+1}\right]}=\frac{\left|\mathbf{k}_{\mathbf{n}+\mathbf{1}}-\mathbf{k}_{\mathbf{n}}\right|}{\left|\mathbf{k}_{\mathbf{n}+\mathbf{1}}\right|} \quad \forall \mathbf{n}=\left(n_{1}, n_{2}, \ldots, n_{d}\right) \geq \mathbf{N}_{0} .
\end{aligned}
$$

Hence

$$
\begin{aligned}
& \left(\frac{\alpha-1}{2 \alpha}\right)^{d} \sum_{\mathbf{n} \geq \mathbf{N}_{0}} \min _{\mathbf{k} \in\left(\mathbf{k}_{\mathbf{n}}, \mathbf{k}_{\mathbf{n}+\mathbf{1}}\right]} \mathrm{P}\left(\left|S_{\mathbf{k}}-B_{\mathbf{k}}\right|>\varepsilon|\mathbf{k}|^{1 / r}\right) \\
& \leq \sum_{\mathbf{n} \geq \mathbf{N}_{0}} \frac{\left|\mathbf{k}_{\mathbf{n}+\mathbf{1}}-\mathbf{k}_{\mathbf{n}}\right|}{\left|\mathbf{k}_{\mathbf{n}+\mathbf{1}}\right|} \min _{\mathbf{k} \in\left(\mathbf{k}_{\mathbf{n}}, \mathbf{k}_{\mathbf{n}+1}\right]} \mathrm{P}\left(\left|S_{\mathbf{k}}-B_{\mathbf{k}}\right|>\varepsilon|\mathbf{k}|^{1 / r}\right) .
\end{aligned}
$$


By this inequality and (3.4), it follows that

$$
\sum_{\mathbf{n} \geq \mathbf{N}_{0}} \min _{\mathbf{k} \in\left(\mathbf{k}_{\mathbf{n}}, \mathbf{k}_{\mathbf{n}+1}\right]} \mathrm{P}\left(\left|S_{\mathbf{k}}-B_{\mathbf{k}}\right|>\varepsilon|\mathbf{k}|^{1 / r}\right)<\infty .
$$

If $\mathbf{n} \geq \mathbf{N}_{0}$ then there exists $\mathbf{m}_{\mathbf{n}} \in \mathbb{N}^{d}$ such that $\mathbf{m}_{\mathbf{n}} \in\left(\mathbf{k}_{\mathbf{n}}, \mathbf{k}_{\mathbf{n}+\mathbf{1}}\right]$ and

$$
\mathrm{P}\left(\left|S_{\mathbf{m}_{\mathbf{n}}}-B_{\mathbf{m}_{\mathbf{n}}}\right|>\varepsilon\left|\mathbf{m}_{\mathbf{n}}\right|^{1 / r}\right)=\min _{\mathbf{k} \in\left(\mathbf{k}_{\mathbf{n}}, \mathbf{k}_{\mathbf{n}+\mathbf{1}}\right]} \mathrm{P}\left(\left|S_{\mathbf{k}}-B_{\mathbf{k}}\right|>\varepsilon|\mathbf{k}|^{1 / r}\right) .
$$

Therefore, by (3.5) we have

$$
\sum_{\mathbf{n} \geq \mathbf{N}_{0}} \mathrm{P}\left(\left|S_{\mathbf{m}_{\mathbf{n}}}-B_{\mathbf{m}_{\mathbf{n}}}\right|>\varepsilon\left|\mathbf{m}_{\mathbf{n}}\right|^{1 / r}\right)<\infty .
$$

By the Borel-Cantelli lemma, (3.6) implies that there exist $\mathbf{N}_{1} \in \mathbb{N}^{d}$ and $A \in \mathcal{F}$ such that $\mathbf{N}_{1} \geq \mathbf{N}_{0}, \mathrm{P}(A)=1$ and

$$
\frac{\left|S_{\mathbf{m}_{\mathbf{n}}}(\omega)-B_{\mathbf{m}_{\mathbf{n}}}\right|}{\left|\mathbf{m}_{\mathbf{n}}\right|^{1 / r}} \leq \varepsilon \quad \forall \mathbf{n} \geq \mathbf{N}_{1}, \forall \omega \in A .
$$

Henceforward let $\omega \in A$ be fixed.

If $\mathbf{n} \geq \mathbf{N}_{1}$ and $\mathbf{t} \in\left(\mathbf{k}_{\mathbf{n}+\mathbf{1}}, \mathbf{k}_{\mathbf{n}+\mathbf{2}}\right]$, then by $\mathbf{t} \in\left(\mathbf{m}_{\mathbf{n}}, \mathbf{m}_{\mathbf{n}+\mathbf{2}}\right],(3.7)$ and

$$
\left|\mathbf{m}_{\mathbf{n}+\mathbf{2}}\right|^{1 / r} \geq\left|\mathbf{m}_{\mathbf{n}}\right|^{1 / r} \geq\left|\mathbf{m}_{\mathbf{n}}\right|
$$

we have

$$
\begin{aligned}
\frac{S_{\mathbf{t}}(\omega)-B_{\mathbf{t}}}{|\mathbf{t}|^{1 / r}} & \geq \frac{S_{\mathbf{m}_{\mathbf{n}}}(\omega)-B_{\mathbf{m}_{\mathbf{n}+\mathbf{2}}}}{\left|\mathbf{m}_{\mathbf{n}+\mathbf{2}}\right|^{1 / r}} \\
& =\frac{S_{\mathbf{m}_{\mathbf{n}}}(\omega)-B_{\mathbf{m}_{\mathbf{n}}}}{\left|\mathbf{m}_{\mathbf{n}}\right|^{1 / r}} \frac{\left|\mathbf{m}_{\mathbf{n}}\right|^{1 / r}}{\left|\mathbf{m}_{\mathbf{n}+\mathbf{2}}\right|^{1 / r}}-\frac{B_{\mathbf{m}_{\mathbf{n}+\mathbf{2}}}-B_{\mathbf{m}_{\mathbf{n}}}}{\left|\mathbf{m}_{\mathbf{n}+\mathbf{2}}\right|^{1 / r}} \\
& \geq-\varepsilon-\frac{B_{\mathbf{m}_{\mathbf{n}+\mathbf{2}}}-B_{\mathbf{m}_{\mathbf{n}}}}{\left|\mathbf{m}_{\mathbf{n}}\right|}
\end{aligned}
$$

If $\mathbf{n}=\left(n_{1}, n_{2}, \ldots, n_{d}\right) \geq \mathbf{N}_{0}$ and $\mathbf{m}_{\mathbf{n}}=\left(\mathbf{m}_{\mathbf{n}}^{(1)}, \mathbf{m}_{\mathbf{n}}^{(2)}, \ldots, \mathbf{m}_{\mathbf{n}}^{(d)}\right)$ then

$$
\left[\alpha^{n_{i}}\right]<\mathbf{m}_{\mathbf{n}}^{(i)} \leq\left[\alpha^{n_{i}+1}\right] .
$$

On the other hand $\mathbf{m}_{\mathbf{n}}^{(i)} \in \mathbb{N}$, hence we get

$$
\alpha^{n_{i}}<\mathbf{m}_{\mathbf{n}}^{(i)} \leq \alpha^{n_{i}+1} .
$$

This inequality implies

$$
\left|\mathbf{m}_{\mathbf{n}+\mathbf{2}}\right|-\left|\mathbf{m}_{\mathbf{n}}\right|>\prod_{i=1}^{d} \alpha^{n_{i}+2}-\prod_{i=1}^{d} \alpha^{n_{i}+1}
$$




$$
\begin{aligned}
& =\left(\alpha^{d}-1\right) \prod_{i=1}^{d} \alpha^{n_{i}+1} \\
& >\left(\alpha^{d}-1\right) \alpha^{n_{1}} \quad \forall \mathbf{n}=\left(n_{1}, n_{2}, \ldots, n_{d}\right) \geq \mathbf{N}_{0} .
\end{aligned}
$$

Since $\lim _{n \rightarrow \infty} \alpha^{n}=\infty$, therefore $\alpha^{n} \geq K\left(\alpha^{d}-1\right)^{-1}$ except for finitely many values of $n \in \mathbb{N}$. Hence there exists $\mathbf{N}_{2} \in \mathbb{N}^{\bar{d}}$ such that $\mathbf{N}_{2} \geq \mathbf{N}_{1}$ and

$$
\left|\mathbf{m}_{\mathbf{n}+\mathbf{2}}\right|-\left|\mathbf{m}_{\mathbf{n}}\right|>\left(\alpha^{d}-1\right) \frac{K}{\alpha^{d}-1}=K \quad \forall \mathbf{n} \geq \mathbf{N}_{2} .
$$

This inequality implies by (3.1), that

$$
B_{\mathbf{m}_{\mathbf{n}+\mathbf{2}}}-B_{\mathbf{m}_{\mathbf{n}}} \leq c\left(\left|\mathbf{m}_{\mathbf{n}+\mathbf{2}}\right|-\left|\mathbf{m}_{\mathbf{n}}\right|\right) \quad \forall \mathbf{n} \geq \mathbf{N}_{2} .
$$

Using (3.9) we have

$$
\frac{\left|\mathbf{m}_{\mathbf{n}+\mathbf{2}}\right|}{\left|\mathbf{m}_{\mathbf{n}}\right|} \leq \prod_{i=1}^{d} \frac{\alpha^{n_{i}+3}}{\alpha^{n_{i}}}=\alpha^{3 d} \quad \forall \mathbf{n}=\left(n_{1}, n_{2}, \ldots, n_{d}\right) \geq \mathbf{N}_{2} .
$$

Hence (3.8), (3.10), (3.11) and (3.3) imply, that if $\mathbf{n} \geq \mathbf{N}_{2}$ and $\mathbf{t} \in\left(\mathbf{k}_{\mathbf{n}+\mathbf{1}}, \mathbf{k}_{\mathbf{n}+\mathbf{2}}\right]$, then

$$
\begin{aligned}
\frac{S_{\mathbf{t}}(\omega)-B_{\mathbf{t}}}{|\mathbf{t}|^{1 / r}} & \geq-\varepsilon-\frac{B_{\mathbf{m}_{\mathbf{n}+\mathbf{2}}}-B_{\mathbf{m}_{\mathbf{n}}}}{\left|\mathbf{m}_{\mathbf{n}}\right|} \geq-\varepsilon-c\left(\frac{\left|\mathbf{m}_{\mathbf{n}+\mathbf{2}}\right|}{\left|\mathbf{m}_{\mathbf{n}}\right|}-1\right) \\
& \geq-\varepsilon-c\left(\alpha^{3 d}-1\right) \geq-\varepsilon \alpha^{3 d / r}-c\left(\alpha^{3 d}-1\right)>-\delta .
\end{aligned}
$$

If $\mathbf{n} \geq \mathbf{N}_{2}$ and $\mathbf{t} \in\left(\mathbf{k}_{\mathbf{n}+\mathbf{1}}, \mathbf{k}_{\mathbf{n}+\mathbf{2}}\right]$, then by $\mathbf{t} \in\left(\mathbf{m}_{\mathbf{n}}, \mathbf{m}_{\mathbf{n}+\mathbf{2}}\right],\left|\mathbf{m}_{\mathbf{n}}\right|^{1 / r} \geq\left|\mathbf{m}_{\mathbf{n}}\right|,(3.7)$, (3.11), (3.10) and (3.3), we have

$$
\begin{aligned}
\frac{S_{\mathbf{t}}(\omega)-B_{\mathbf{t}}}{|\mathbf{t}|^{1 / r}} & \leq \frac{S_{\mathbf{m}_{\mathbf{n}+\mathbf{2}}}(\omega)-B_{\mathbf{m}_{\mathbf{n}}}}{\left|\mathbf{m}_{\mathbf{n}}\right|^{1 / r}} \\
& =\frac{S_{\mathbf{m}_{\mathbf{n}+\mathbf{2}}}(\omega)-B_{\mathbf{m}_{\mathbf{n}+\mathbf{2}}}}{\left|\mathbf{m}_{\mathbf{n}+\mathbf{2}}\right|^{1 / r}} \frac{\left|\mathbf{m}_{\mathbf{n}+\mathbf{2}}\right|^{1 / r}}{\left|\mathbf{m}_{\mathbf{n}}\right|^{1 / r}}+\frac{B_{\mathbf{m}_{\mathbf{n}+\mathbf{2}}}-B_{\mathbf{m}_{\mathbf{n}}}}{\left|\mathbf{m}_{\mathbf{n}}\right|^{1 / r}} \\
& \leq \frac{S_{\mathbf{m}_{\mathbf{n}+\mathbf{2}}}(\omega)-B_{\mathbf{m}_{\mathbf{n}+\mathbf{2}}}}{\left|\mathbf{m}_{\mathbf{n}+\mathbf{2}}\right|^{1 / r}} \frac{\left|\mathbf{m}_{\mathbf{n}+\mathbf{2}}\right|^{1 / r}}{\left|\mathbf{m}_{\mathbf{n}}\right|^{1 / r}}+\frac{B_{\mathbf{m}_{\mathbf{n}+\mathbf{2}}}-B_{\mathbf{m}_{\mathbf{n}}}}{\left|\mathbf{m}_{\mathbf{n}}\right|} \\
& \leq \varepsilon \alpha^{3 d / r}+c\left(\frac{\left|\mathbf{m}_{\mathbf{n}+\mathbf{2}}\right|}{\left|\mathbf{m}_{\mathbf{n}}\right|}-1\right) \leq \varepsilon \alpha^{3 d / r}+c\left(\alpha^{3 d}-1\right)<\delta .
\end{aligned}
$$

This inequality and (3.12) imply

$$
\frac{\left|S_{\mathbf{t}}(\omega)-B_{\mathbf{t}}\right|}{|\mathbf{t}|^{1 / r}}<\delta \quad \forall \mathbf{n} \geq \mathbf{N}_{2}, \mathbf{t} \in\left(\mathbf{k}_{\mathbf{n}+\mathbf{1}}, \mathbf{k}_{\mathbf{n}+\mathbf{2}}\right] .
$$

If $\mathbf{t} \geq \mathbf{k}_{\mathbf{N}_{2}+\mathbf{1}}+\mathbf{1}$, then there exists $\mathbf{n} \geq \mathbf{N}_{2}$ such that $\mathbf{t} \in\left(\mathbf{k}_{\mathbf{n}+\mathbf{1}}, \mathbf{k}_{\mathbf{n}+\mathbf{2}}\right]$. Hence (3.13) implies

$$
\frac{\left|S_{\mathbf{t}}(\omega)-B_{\mathbf{t}}\right|}{|\mathbf{t}|^{1 / r}}<\delta \quad \forall \mathbf{t} \geq \mathbf{k}_{\mathbf{N}_{2}+\mathbf{1}}+\mathbf{1} .
$$

Therefore the statement is proved. 


\section{An application for multi-index sequence of non- negative random variables with finite variances}

In this section we give an application of Theorem 3.1. In case $d=r=1$, this result gives Theorem of Petrov [9].

Theorem 4.1. Let $X_{\mathbf{n}}\left(\mathbf{n} \in \mathbb{N}^{d}\right)$ be a sequence of non-negative random variables with finite variances, $S_{\mathbf{n}}=\sum_{\mathbf{k} \leq \mathbf{n}} X_{\mathbf{k}}, c>0, K \in \mathbb{N}$ and $0<r \leq 1$. If

$$
\mathrm{E} S_{\mathbf{n}}-\mathrm{E} S_{\mathbf{m}} \leq c(|\mathbf{n}|-|\mathbf{m}|) \quad \forall \mathbf{n}, \mathbf{m} \in \mathbb{N}^{d}, \mathbf{n} \geq \mathbf{m},|\mathbf{n}|-|\mathbf{m}| \geq K
$$

and

$$
\sum_{\mathbf{n}} \frac{\operatorname{Var} S_{\mathbf{n}}}{|\mathbf{n}|^{1+2 / r}}<\infty
$$

then

$$
\lim _{\mathbf{n} \rightarrow \infty} \frac{S_{\mathbf{n}}-\mathrm{E} S_{\mathbf{n}}}{|\mathbf{n}|^{1 / r}}=0 \quad \text { almost surely. }
$$

Proof. With notation $b_{\mathbf{k}}=\mathrm{E} X_{\mathbf{k}}$ and $B_{\mathbf{n}}=\sum_{\mathbf{k}<\mathbf{n}} b_{\mathbf{k}}=\mathrm{E} S_{\mathbf{n}}$, (4.1) implies (3.1). On the other hand, if $\varepsilon>0$, then the Chebyshev inequality and (4.2) imply

$$
\sum_{\mathbf{n}} \frac{1}{|\mathbf{n}|} \mathrm{P}\left(\left|S_{\mathbf{n}}-B_{\mathbf{n}}\right|>\varepsilon|\mathbf{n}|^{1 / r}\right) \leq \sum_{\mathbf{n}} \frac{1}{|\mathbf{n}|} \frac{\operatorname{Var} \frac{S_{\mathbf{n}}}{|\mathbf{n}|^{1 / r}}}{\varepsilon^{2}}=\varepsilon^{-2} \sum_{\mathbf{n}} \frac{\operatorname{Var} S_{\mathbf{n}}}{|\mathbf{n}|^{1+2 / r}}<\infty .
$$

Therefore (3.2) holds. Hence, using Theorem 3.1, we have that the statement is true.

\section{References}

[1] N. Etemadi: An elementary proof of the strong law of large numbers, Z. Wahrscheinlichkeitstheorie Verw. Gebiete 55.1 (1981), pp. 119-122, DOI: 10.1007/bf01013465.

[2] I. Fazekas: Convergence rates in the Marcinkiewicz strong law of large numbers for Banach space valued random variables with multidimensional indices, Publicationes Mathematicae, Debrecen 32 (1985), pp. 203-209.

[3] I. FAzekas, T. TómÁcs: Strong laws of large numbers for pairwise independent random variables with multidimensional indices, Publicationes Mathematicae, Debrecen 53.1-2 (1998), pp. 149-161.

[4] A. Gut: Marcinkiewicz laws and convergence rates in the law of large numbers for random variables with multidimensional indices, The Annals of Probability 6.3 (1978), pp. 469-482, DOI: $10.1214 / \mathrm{aop} / 1176995531$.

[5] O. I. KLesov: Strong law of large numbers for random fields with orthogonal values, Dokl. Akad. Nauk. Ukr. SSR Ser. A 7 (1982), pp. 9-12.

[6] O. I. KLesov: The law of large numbers for multiple sums of independent identically distributed random variables, Theor. Probab. Math. Statist. 50 (1995), pp. 77-87. 
[7] V. M. Kruglov: Strong law of large numbers, in: Stability Problems for Stochastic Models: Proceedings of the Fifteenth Perm Seminar, Perm, Russia, June 2-6, 1992, Moscow, Utrecht, Tokyo: TVP/VSP, 1994, pp. 139-150, ISBN: 90-6764-159-6.

[8] M. LoÈve: Probability Theory I. New York: Springer-Verlag, 1977.

[9] V. V. Petrov: On the strong law of large numbers for a sequence of nonnegative random variables, Zapiski Nauchnnykh Seminarov POMI 384 (2010), pp. 182-184, DOI: $10.1007 /$ s10958-011-0411-x. 